\title{
BOUNDED WEAK SOLUTIONS TO ELLIPTIC PDE WITH DATA IN ORLICZ SPACES
}

\author{
DAVID CRUZ-URIBE, OFS AND SCOTT RODNEY
}

\begin{abstract}
A classical regularity result is that non-negative solutions to the Dirichlet problem $\Delta u=f$ in a bounded domain $\Omega$, where $f \in L^{q}(\Omega), q>\frac{n}{2}$, satisfy $\|u\|_{L^{\infty}(\Omega)} \leq C\|f\|_{L^{q}(\Omega)}$. We extend this result in three ways: we replace the Laplacian with a degenerate elliptic operator; we show that we can take the data $f$ in an Orlicz space $L^{A}(\Omega)$ that lies strictly between $L^{\frac{n}{2}}(\Omega)$ and $L^{q}(\Omega), q>\frac{n}{2}$; and we show that that we can replace the $L^{A}$ norm in the right-hand side by a smaller expression involving the logarithm of the "entropy bump" $\|f\|_{L^{A}(\Omega)} /\|f\|_{L^{\frac{n}{2}(\Omega)}}$, generalizing a result due to $\mathrm{Xu}$.
\end{abstract}

\section{IntRODUCTION}

In this paper, we consider boundedness properties of weak (sub)solutions to the following Dirichlet problem:

$$
\left\{\begin{aligned}
-\operatorname{Div}(Q \nabla u) & =f v & & \text { for } x \in \Omega \\
u & =0 & & \text { for } x \in \partial \Omega
\end{aligned}\right.
$$

Throughout this paper, $\Omega$ is a bounded domain (i.e., an open and connected subset) of $\mathbb{R}^{n}$ with $n \geq 3, Q=Q(x)$ is a non-negative definite, symmetric, measurable matrix with $Q \in L_{l o c}^{1}(\Omega), v$ is a weight (i.e., a non-negative, measurable function) on $\Omega$ such that $v \in L^{1}(\Omega)$, and the data function $f$ is in $L_{l o c}^{1}(\Omega)$.

When $Q$ is a uniformly elliptic matrix and $v(x)=1$, it is a classical result (See Maz'ya [16, 17], Stampacchia [23] and Trudinger [25, Theorem 4.1], [12, Theorem 8.16]) that there is a constant $C>0$ such that if $f \in L^{q}(\Omega)$, then

$$
\|u\|_{L^{\infty}(\Omega)} \leq C\|f\|_{L^{q}(\Omega)}
$$

Date: November 29, 2020.

2020 Mathematics Subject Classification. 35B45, 35D30, 35J25, 46E30.

Key words and phrases. Orlicz spaces, degenerate elliptic equations, bounded solutions, a priori estimates.

D. Cruz-Uribe is supported by research funds from the Dean of the College of Arts \& Sciences, the University of Alabama. S. Rodney is supported by the NSERC Discovery Grant program. The authors would like to thank Andrea Cianchi for calling to our attention several important references. 
for any non-negative weak subsolution $u \in H^{1}(\Omega)$ of $(1.1)$ provided $q>\frac{n}{2}$. Moreover, a counter-example shows that this bound is sharp even for the Laplacian and we cannot take $q=\frac{n}{2}$.

The standard proof of this result uses the classical Sobolev inequality,

$$
\|\psi\|_{L^{\frac{2 n}{n-2}(\Omega)}} \leq C\|\nabla \psi\|_{L^{2}(\Omega)},
$$

valid for any $\psi \in H_{0}^{1}(\Omega)$, combined with Moser iteration. The restriction $q>\frac{n}{2}$ is naturally connected to the classical Sobolev gain factor $\sigma=\frac{n}{n-2}=\left(\frac{n}{2}\right)^{\prime}$.

The goal of this paper is to generalize this result. First we show that this estimate can be improved by replacing the space $L^{q}(\Omega)$ by an Orlicz space $L^{A}(\Omega)$ that lies strictly between $L^{\frac{n}{2}}(\Omega)$ and $L^{q}(\Omega)$ for $q>\frac{n}{2}$. For brevity, we will defer many definitions to Section 2 below.

Theorem 1.2. Let $Q$ be a uniformly elliptic matrix, and let $f \in L^{A}(\Omega)$, where $A(t)=t^{\frac{n}{2}} \log (e+t)^{q}, q>\frac{n}{2}$. Then there exists a constant $C=C(n, q, Q)$ such that, given any non-negative weak subsolution $u$ of

$$
\left\{\begin{aligned}
-\operatorname{Div}(Q \nabla u) & =f \quad \text { for } x \in \Omega \\
u & =0 \quad \text { for } x \in \partial \Omega
\end{aligned}\right.
$$

we have the estimate

$$
\|u\|_{L^{\infty}(\Omega)} \leq C\|f\|_{L^{A}(\Omega)}
$$

Remark 1.3. After completing this paper we learned that a somewhat more general version of Theorem 1.2 was proved by Cianchi [4, Theorem 5] using very different methods.

We will prove Theorem 1.2 as a special case of a more general result for solutions of (1.1) that holds for a much larger class of matrices $Q$. We can allow $Q$ to be both degenerate and singular, but must impose some restrictions on the largest and smallest eigenvalues. We encode these restrictions in two assumptions on the integrability of the largest eigenvalue and on the existence of an $L^{2}$ Sobolev inequality with gain. We state them together as a general hypothesis.

Hypothesis 1.4. Given the matrix $Q$ and the weight $v \in L^{1}(\Omega)$, assume that for some constant $k>0$,

$$
|Q(x)|_{\text {op }}=\sup \left\{|Q(x) \xi|: \xi \in \mathbb{R}^{n},|\xi|=1\right\} \leq k v(x) \text { a.e. }
$$

Moreover, assume that there exist constants $\sigma=\sigma(n, Q, v, \Omega)>1, C_{0} \geq 1$ such that for every $\psi \in \operatorname{Lip}_{0}(\Omega)$

$$
\left(\int_{\Omega}|\psi(x)|^{2 \sigma} v(x) d x\right)^{\frac{1}{2 \sigma}} \leq C_{0}\left(\int_{\Omega}|\sqrt{Q(x)} \nabla \psi(x)|^{2} d x\right)^{\frac{1}{2}} .
$$


The first assumption, that $|Q|_{o p} \leq k v$, in Hypothesis 1.4 is necessary to prove many of the necessary properties of weak derivatives in the corresponding degenerate Sobolev space. The second assumption, that inequality (1.5) holds, in Hypothesis 1.4 reduces to the classical Sobolev inequality if $Q$ is a uniformly elliptic matrix in $\Omega$ and $\sigma=\frac{n}{n-2}=\left(\frac{n}{2}\right)^{\prime}$. It allows us to perform the necessary De Giorgi iteration.

These assumptions hold in several important special cases. If $v$ satisfies the Muckenhoupt $A_{2}$ condition,

$$
[v]_{A_{2}}=\sup _{B} \frac{1}{|B|} \int_{B} v(x) d x \frac{1}{|B|} \int_{B} v(x)^{-1} d x<\infty,
$$

where the supremum is taken over all balls $B$ in $\mathbb{R}^{n}$, and if $Q$ satisfies the degenerate ellipticity condition

$$
\lambda v(x)|\xi|^{2} \leq\langle Q(x) \xi, \xi\rangle \leq \Lambda v(x)|\xi|^{2},
$$

where $0<\lambda \leq \Lambda<\infty$, then $|Q|_{o p} \leq \Lambda v$ and (1.5) holds. (See [10].) More generally, suppose that $u$ and $v$ are a pair of weights such that $u(x) \leq v(x)$ a.e., $v$ satisfies a doubling condition, $u \in A_{2}$, and there exists $\sigma>1$ such that given any balls $B_{1} \subset B_{2} \subset \Omega$,

$$
\frac{r\left(B_{1}\right)}{r\left(B_{2}\right)}\left(\frac{v\left(B_{1}\right)}{v\left(B_{2}\right)}\right)^{\frac{1}{2 \sigma}} \leq C\left(\frac{u\left(B_{1}\right)}{u\left(B_{2}\right)}\right)^{\frac{1}{2}},
$$

and $Q$ satisfies the degenerate ellipticity condition

$$
u(x)|\xi|^{2} \leq\langle Q(x) \xi, \xi\rangle \leq v(x)|\xi|^{2},
$$

then $|Q|_{o p} \leq v$ and we have the Sobolev inequality

$$
\left(\int_{\Omega}|\psi(x)|^{2 \sigma} v(x) d x\right)^{\frac{1}{2 \sigma}} \leq C_{0}\left(\int_{\Omega}|\nabla \psi(x)|^{2} u(x) d x\right)^{\frac{1}{2}}
$$

so again (1.5) holds. (See [1].)

Remark 1.6. In [7], the authors and Rosta proved that when $v=1$, with minor additional hypotheses the global Sobolev inequality (1.5) follows from a weaker, local Sobolev inequality,

$$
\left(\frac{1}{|B|} \int_{B}|\psi(x)|^{2 \sigma} d x\right)^{\frac{1}{2 \sigma}} \leq C\left[\frac{r(B)}{|B|} \int_{B}|\sqrt{Q} \nabla \psi(x)|^{2} d x+\frac{1}{|B|} \int_{B}|\psi(x)|^{2} d x\right]^{\frac{1}{2}}
$$

that holds for all (sufficiently small) balls $B \subset \Omega$.

We can now state our main result, which is a generalization of Theorem 1.2 to degenerate elliptic operators. Again, for precise definitions see Section 2. 
Theorem 1.7. Given a weight $v$ and the non-negative definite, symmetric matrix $Q$, suppose that Hypothesis 1.4 holds for some $\sigma>1$. Let $A(t)=t^{\sigma^{\prime}} \log (e+t)^{q}$ where $q>\sigma^{\prime}$. If $f \in L^{A}(v ; \Omega)$, then any non-negative weak subsolution $\mathbf{u}=(u, \nabla u) \in$ $Q H_{0}^{1}(v ; \Omega)$ of $(1.1)$ satisfies

$$
\|u\|_{L^{\infty}(v ; \Omega)} \leq C\|f\|_{L^{A}(v ; \Omega)}
$$

where $C$ is independent of both $\mathbf{u}$ and $f$.

We originally conjectured that the exponent $q$ in Theorem 1.7 is sharp in general, but we were not able to prove this or find a counter-example. We then learned that Cianchi [4, Theorem 5], as a consequence of a more general result, showed that in the classical setting when $Q$ is uniformly elliptic and $\sigma=\left(\frac{n}{2}\right)^{\prime}$, we must have that $q>\frac{n}{2}-1$. We round out his result by giving a simple counter-example proving that it is sharp for the Laplacian.

Example 1.9. Let $n \geq 3$, and let $\Omega=B(0,1)$. Then there exists a function $f \in$ $L^{A}(\Omega)$, where $A(t)=t^{\frac{n}{2}} \log (e+t)^{q}, q<\frac{n}{2}-1$, such that the non-negative weak solution of the Poisson equation

$$
\left\{\begin{aligned}
-\Delta u=f & \text { for } x \in \Omega \\
u & =0 \quad \text { for } x \in \partial \Omega
\end{aligned}\right.
$$

is unbounded.

Remark 1.10. We conjecture that the sharp exponent in Theorem 1.7 is $q>\sigma^{\prime}-1$. However, the bound $q>\sigma^{\prime}$ appears to be intrinsic to our proof, so either our proof needs to be refined or another approach is needed. We note that the proof in [4] relies on re-arrangement estimates and so does not readily extend to the case of degenerate operators.

Our second main result shows that inequality (1.8) can be sharpened so that the right-hand side only depends on the logarithm of the $L^{A}$ norm.

Theorem 1.11. Given a weight $v$ and a non-negative definite, symmetric matrix $Q$, suppose that Hypothesis 1.4 holds for some $\sigma>1$. Let $A(t)=t^{\sigma^{\prime}} \log (e+t)^{q}$, where $q>\sigma^{\prime}$. If $f \in L^{A}(v ; \Omega)$, then any non-negative weak subsolution $\mathbf{u}=(u, \nabla u) \in$ $Q H_{0}^{1}(v ; \Omega)$ of $(1.1)$ satisfies

$$
\|u\|_{L^{\infty}(v ; \Omega)} \leq C\|f\|_{L^{\sigma^{\prime}(v ; \Omega)}}\left(1+\log \left(1+\frac{\|f\|_{L^{A}(v ; \Omega)}}{\|f\|_{L^{\sigma^{\prime}}(v ; \Omega)}}\right)\right),
$$

where $C$ is independent of both $\mathbf{u}$ and $f$. 
Theorem 1.11 generalizes the main result of $\mathrm{Xu}[26]$, but we note that there is a mistake in the statement of his main result. Working in the same setting as Theorem 1.2, he claims to show that

$$
\|u\|_{L^{\infty}(\Omega)} \leq C\|f\|_{L^{\frac{n}{2}(\Omega)}}\left(1+\log \left(1+\|f\|_{L^{q}(\Omega)}\right)\right),
$$

where $q>\frac{n}{2}$. However, a close examination of his proof shows that he only proves this when $\|f\|_{L^{\frac{n}{2}(\Omega)}} \geq 1$, and in fact what he proves is the analog of Theorem 1.11. It is straightforward to see that (1.13) cannot hold if $\|f\|_{L^{\frac{n}{2}(\Omega)}}<1$; if it did, then if we fix $f$ and the corresponding solution $u$, then we could apply this inequality to $f / N(N>1$ large $)$ and $u / N$. Then we could take the limit as $N \rightarrow \infty$ to conclude that $\|u\|_{L^{\infty}(\Omega)} \leq C\|f\|_{L^{\frac{n}{2}(\Omega)}}$, which is false in general.

Remark 1.14. The ratio $\|f\|_{L^{A}(v ; \Omega)} /\|f\|_{L^{\sigma^{\prime}(v ; \Omega)}}$ in Theorem 1.11 measures how much bigger the Orlicz norm is than the associated Lebesgue space norm. It is similar in spirit, though not in detail, to the "entropy bump" conditions introduced in the study of weighted norm inequalities in harmonic analysis [15, 24].

Our two main results are established via De Giorgi iteration on the level sets. De Giorgi's original arguments are in [8] but more helpful descriptions are found in [2] and in [13], where De Giorgi iteration is applied in an infinitely degenerate elliptic regime. We were unable to adapt Moser iteration to work in the context of Orlicz norms, and it remains an open question whether such an approach is possible in this setting.

The remainder of the paper is organized as follows. In Section 2 we gather some preliminary results. We give a definition of Young functions and the associated Orlicz spaces, and record some useful properties. We then define weak solutions to the Dirichlet problem. This definition has to include the possibility that the matrix $Q$ can be both degenerate and singular, and we give it in terms of a degenerate Sobolev space, building upon results in [6] and elsewhere. We prove a number of properties of weak derivatives in this setting; we believe these results should be useful tools for other problems. We also prove that bounded, non-negative subsolutions of (1.1) must satisfy an exponential integrability condition. This result is a key lemma for the proof of Theorem 1.11 and is modeled on a similar result due to $\mathrm{Xu}[26]$ in the classical setting. For completeness we include the details of the proof. In Section 3 we prove Theorem 1.7; as noted above, the proof uses a version of De Giorgi iteration adapted to the scale of Orlicz spaces. This iteration argument was gotten by a careful adaptation of an argument due Korbenko, et al. [13, Section 4.2]. In Section 4 we prove Theorem 1.11; our proof is a generalization of the argument in [26] and requires us to deal with a number of technical obstacles. Finally, in Section 5 we construct Example 1.9. 


\section{Preliminaries}

In this section we gather some preliminary definitions and results. We begin with some notation. The constant $n$ will always denote the dimension of the underlying space $\mathbb{R}^{n}$. By $C, c$, etc. we will mean a constant that may change from appearance to appearance, but whose value depends only on the underlying parameters. If we want to specify this dependence, we will write, for instance, $C(n, p)$, etc. If we write $A \lesssim B$, we mean that there exists a constant $c$ such that $A \leq c B$. If $A \lesssim B$ and $B \lesssim A$, we write $A \approx B$.

A weight $v$ will always be a non-negative, measurable function such that $v \in L^{1}(\Omega)$. Given a set $E \subset \Omega, v(E)=\int_{E} v(x) d x$. Given a weight $v, L^{p}(v ; \Omega)$ is the collection of all those measurable functions $g: \Omega \rightarrow \mathbb{R}$ for which

$$
\|g\|_{p}=\|g\|_{L^{p}(v ; \Omega)}=\left(\int_{\Omega}|g(x)|^{p} v(x) d x\right)^{1 / p}<\infty .
$$

Orlicz spaces. Our main hypothesis on the data function $f$ in (1.1) is that it belongs to the Orlicz space $L^{A}(v ; \Omega)$. Here we gather some essential results about these spaces but we assume the reader has some familiarity with them. For complete information, we refer to $[14,20]$. For a briefer summary, see [5, Chapter 5].

By a Young function we mean a function $A:[0, \infty) \rightarrow[0, \infty)$ that is continuous, convex, strictly increasing, $A(0)=0$, and $\frac{A(t)}{t} \rightarrow \infty$ as $t \rightarrow \infty$. Given a Young function $A$, define $L^{A}(v ; \Omega)$ to be the Banach space of measurable functions $h: \Omega \rightarrow \mathbb{R}$ equipped with the Luxembourg norm,

$$
\|h\|_{A}=\|h\|_{L^{A}(v ; \Omega)}=\inf \left\{\lambda>0: \int_{\Omega} A\left(\frac{|f(x)|}{\lambda}\right) v(x) d x \leq 1\right\}<\infty .
$$

Given Young functions $A, B$ we can compare the associated norms by appealing to a point-wise estimate. We say that $A(t) \preceq B(t)$ if there is a $t_{0}>0$ and a constant $c \geq 1$ depending only on $A, B$ so that $A(t) \leq B(c t)$ for $t \geq t_{0}$. For a proof of the following result, see [14, Theorem 13.3] or [20, Section 5.1].

Lemma 2.1. Given Young functions $A, B$, if $A \preceq B$, then there exists a constant $C=C(A, B, v(\Omega))$ such that for every $f \in L^{B}(v ; \Omega)$,

$$
\|f\|_{L^{A}(v ; \Omega)} \leq C\|f\|_{L^{B}(v ; \Omega)}
$$

Given a Young function $A$, we define the conjugate Orlicz function, $\bar{A}$, via the pointwise formula

$$
\bar{A}(t)=\sup \{s t-B(s): s>0\} .
$$


The pair $A, \bar{A}$ satisfy a version of Hölder's inequality in the scale of Orlicz spaces. If $f \in L^{A}(v ; \Omega)$ and $g \in L^{\bar{A}}(v ; \Omega)$, then $f g \in L^{1}(v ; \Omega)$ and

$$
\int_{\Omega}|f(x) g(x)| v(x) d x \leq 2\|f\|_{A}\|g\|_{\bar{A}}
$$

In our main results we consider Young functions of the form

$$
B(t)=t^{p} \log (e+t)^{q},
$$

where $1<p, q<\infty$. The inverse and conjugate functions associated with these Young functions are well-known: see, for instance, [5]. We have that

$$
\begin{gathered}
\bar{B}(t) \approx \frac{t^{p^{\prime}}}{\log (t)^{q}} \approx \frac{t^{p^{\prime}}}{\log (e+t)^{q}}, \\
\bar{B}^{-1}(t) \approx t^{1 / p^{\prime}} \log (e+t)^{\frac{q}{p}},
\end{gathered}
$$

where the implicit constants depend on $p, q$. As a consequence of Lemma 2.1 we have the following estimate which we will need below; details are straightforward and are omitted.

Lemma 2.6. Let $1 \leq p_{1} \leq p_{2}<\infty, 1 \leq q_{1} \leq q_{2}<\infty$ and define

$$
A(t)=t^{p_{1}} \log (e+t)^{q_{1}}, \quad B(t)=t^{p_{2}} \log (e+t)^{q_{2}} .
$$

Then, given $f \in L^{B}(v ; \Omega)$,

$$
\|f\|_{L^{p_{1}(v, \Omega)}} \lesssim\|f\|_{L^{A}(v ; \Omega)} \lesssim\|f\|_{L^{p_{2}(v ; \Omega)}} \lesssim\|f\|_{L^{B}(v ; \Omega)} .
$$

The implicit constants depend on $p_{i}$ and $q_{i}, i=1,2$, and $v(\Omega)$.

We conclude this section with an estimate for the $L^{\bar{B}}(\Omega)$ norm of an indicator function $\mathbb{1}_{S}$ for $S \subset \Omega$; this quantity plays an essential role in our proofs of Theorems 1.7 and 1.11. This computation is well-known, but to make clear the dependence on the constants we include its short proof.

Lemma 2.7. Given the Young function $B$ defined by (2.3) then for any $S \subset \Omega$, $v(S)>0$,

$$
\left\|\mathbb{1}_{S}\right\|_{L^{\bar{B}}(v ; \Omega)} \leq \frac{c v(S)^{\frac{1}{p^{\prime}}}}{\log \left(1+v(S)^{-1}\right)^{\frac{q}{p}}}
$$

where $c=c(p, q)>0$.

Proof. Given $B, \bar{B}$ is defined by (2.4). Set

$$
F=\left\{\lambda>0: \int_{\Omega} \bar{B}\left(\frac{\mathbb{1}_{S}(x)}{\lambda}\right) v(x) d x \leq 1\right\}
$$


and notice that $F \neq \emptyset$. For each $\lambda \in F$,

$$
v(S) \bar{B}\left(\frac{1}{\lambda}\right)=\int_{\Omega} \bar{B}\left(\frac{\mathbb{1}_{S}(x)}{\lambda}\right) v(x) d x \leq 1 .
$$

Since $\bar{B}$ is invertible and increasing,

$$
\lambda \geq\left[\bar{B}^{-1}\left(\frac{1}{v(S)}\right)\right]^{-1}=m_{0}>0 .
$$

Again by the invertibility of $\bar{B}$,

$$
\int_{\Omega} \bar{B}\left(\frac{\mathbb{1}_{S}(x)}{m_{0}}\right) v(x) d x=v(S) \bar{B}\left(m_{0}^{-1}\right)=1 .
$$

Hence, $m_{0} \in F$, and it follows that $\left\|\mathbb{1}_{S}\right\|_{L^{\bar{B}}(\Omega)}=m_{0}$. By inequality $(2.5)$,

$$
m_{0}=\bar{B}^{-1}\left(\frac{1}{v(S)}\right) \geq c(p, q) v(S)^{-\frac{1}{p^{\prime}}} \log \left(e+v(S)^{-1}\right)^{\frac{q}{p}}
$$

and (2.8) follows.

Degenerate Sobolev spaces and weak solutions. We now give a precise definition of weak (sub)solutions to the Dirichlet problem (1.1). This question has been explored in a number of papers by ourselves and others: see $[3,6,7,12,18,19,21,22]$. Here we sketch the relevant details.

Given a non-negative definite, symmetric and measurable matrix function $Q$ on $\Omega$ and a weight $v \in L_{l o c}^{1}(\Omega)$, the solution space for the Dirichlet problem is the matrix weighted Sobolev space $Q H_{0}^{1}(v ; \Omega)$. This space is defined as the abstract completion (in terms of Cauchy sequences) of the space $\operatorname{Lip}_{0}(\Omega)$ (i.e., Lipschitz functions with compact support in $\Omega$ ) with respect to the norm

$$
\|\psi\|_{Q H_{0}^{1}(v ; \Omega)}=\|\psi\|_{L^{2}(v ; \Omega)}+\|\nabla \psi\|_{L_{Q}^{2}(\Omega)},
$$

where $L_{Q}^{2}(\Omega)$ is the Banach space of $\mathbb{R}^{n}$ vector-valued functions $\mathbf{g}$ on $\Omega$ that satisfy

$$
\|\mathbf{g}\|_{L_{Q}^{2}(\Omega)}=\left(\int_{\Omega}|\sqrt{Q(x)} \mathbf{g}(x)|^{2} d x\right)^{\frac{1}{2}}<\infty .
$$

This norm is well defined for $\psi \in \operatorname{Lip}_{0}(\Omega)$ provided $|Q|_{o p} \in L_{l o c}^{1}(\Omega)$; in particular, $Q H_{0}^{1}(v ; \Omega)$ is well defined if the first assumption in Hypothesis 1.4 holds.

With this definition, the Sobolev space $Q H_{0}^{1}(v ; \Omega)$ is a collection of equivalence classes of Cauchy sequences of $\operatorname{Lip}_{0}(\Omega)$ functions. However, the spaces $L^{2}(v ; \Omega)$ and $L_{Q}^{2}(\Omega)$ are complete: for a proof that $L_{Q}^{2}(\Omega)$ is complete, see [22] or [6] where it was proved that $L_{Q}^{p}(\Omega)$ is complete for $1 \leq p<\infty$. Therefore, to each equivalence class 
$\left[\left\{\psi_{j}\right\}\right]$ in $Q H_{0}^{1}(v ; \Omega)$ we can associate a unique pair $\mathbf{u}=(u, \mathbf{g}) \in L^{2}(v ; \Omega) \times L_{Q}^{2}(\Omega)$ whose norm is given by

$$
\begin{aligned}
\|\mathbf{u}\|_{Q H_{0}^{1}(v ; \Omega)} & =\|u\|_{L^{2}(v ; \Omega)}+\|\mathbf{g}\|_{L_{Q}^{2}(\Omega)} \\
& =\lim _{j \rightarrow \infty}\left(\left\|\psi_{j}\right\|_{L^{2}(v ; \Omega)}+\left\|\nabla \psi_{j}\right\|_{L_{Q}^{2}(\Omega)}\right) .
\end{aligned}
$$

Conversely, given a pair $(u, \mathbf{g})$ we will say that it is in $Q H_{0}^{1}(v ; \Omega)$ if there exists a sequence $\left\{u_{j}\right\}_{j} \subset \operatorname{Lip}_{0}(\Omega)$ such that $\left(u_{j} \nabla u_{j}\right)$ converges to $(u, \mathbf{g})$ in $L^{2}(v ; \Omega) \times L_{Q}^{2}(\Omega)$.

Hereafter, we will denote $\mathbf{g}$ by $\nabla u$ since $\mathbf{g}$ plays the role of a weak gradient of $u$. However, while we adopt this formal notation we want to stress that the function $\mathbf{g}$ is not the weak gradient of $u$ in the sense of classical Sobolev spaces. For further details, [6] contains the construction of $Q H^{1, p}(v ; \Omega)$ for $p \geq 1$. Additionally, unweighted constructions of $Q H_{0}^{1, p}(1 ; \Omega)$ are found in [7, 19] for $p \geq 1$ and $[18,21]$ for $p=2$.

We can extend the second assumption in Hypothesis 1.4 to functions in $Q H_{0}^{1}(\Omega)$; this follows from density of $\operatorname{Lip}_{0}(\Omega)$ functions and we omit the proof.

Lemma 2.9. Suppose the Sobolev inequality of Hypothesis 1.4 holds. Then,

$$
\left(\int_{\Omega}|w(x)|^{2 \sigma} v(x) d x\right)^{\frac{1}{2 \sigma}} \leq C_{0}\left(\int_{\Omega}|\sqrt{Q(x)} \nabla w|^{2} d x\right)^{\frac{1}{2}}
$$

for every $\mathbf{w}=(w, \nabla w) \in Q H_{0}^{1}(v ; \Omega)$ where $C_{0}$ is the same as in Hypothesis 1.4.

We can now define the weak solution to the Dirichlet problem.

Definition 2.11. A pair $\mathbf{u}=(u, \nabla u) \in Q H_{0}^{1}(\Omega)$ is said to be a weak solution of the Dirichlet problem (1.1) if

$$
\int_{\Omega} \nabla \psi(x) \cdot Q(x) \nabla u(x) d x=\int_{\Omega} f(x) \psi(x) v(x) d x
$$

for every $\psi \in \operatorname{Lip}_{0}(\Omega)$. The pair is said to be a non-negative weak subsolution if $u(x) \geq 0$ v-a.e. and

$$
\int_{\Omega} \nabla \psi(x) \cdot Q(x) \nabla u(x) d x \leq \int_{\Omega} f(x) \psi(x) v(x) d x
$$

for every non-negative $\psi \in \operatorname{Lip}_{0}(\Omega)$.

Note that if $\mathbf{h}=(h, \nabla h) \in Q H_{0}^{1}(v ; \Omega)$ with $h(x) \geq 0 v$-a.e., then by a standard limiting argument we may use $h$ as our test function in Definition 2.11.

Remark 2.12. The existence of weak solutions to (1.1) when $v=1$ was studied in $[12,18,21]$, and when $v(x)=|Q(x)|_{\text {op }}$ in $[6,7]$. 
Properties of weak gradients. We now develop some useful properties of functions in the degenerate Sobolev space $Q H_{0}^{1}(v ; \Omega)$. All of these properties are well known in the classical case: see, for instance, [12]. In the degenerate case, we stress that the first assumption in Hypothesis 1.4 is critical in proving these results and throughout this subsection we assume that $v \in L^{1}(\Omega)$ and $|Q|_{o p} \leq k v$ a.e.

Our first result shows that weak gradients are zero almost everywhere on sets of $v$-measure zero.

Lemma 2.13. Let $\mathbf{u}=(u, \nabla u) \in Q H_{0}^{1}(v ; \Omega)$ and $w \in \operatorname{Lip}_{0}(\Omega)$. Then, given any set E of v-measure zero, we have that:

(1) $\|\nabla w\|_{L_{Q}^{2}(E)}=0$;

(2) $\|\nabla u\|_{L_{Q}^{2}(E)}=0$;

(3) $\sqrt{Q(x)} \nabla u(x)=0=\sqrt{Q(x)} \nabla w(x)$ a.e. $x \in E$.

Proof. If $w \in \operatorname{Lip}_{0}(\Omega)$, then $\nabla w$ is defined a.e. in $\Omega$ by the Rademacher-Stepanov theorem and is in $L^{\infty}$. Therefore, for a.e. $x \in \Omega$,

$$
|\sqrt{Q(x)} \nabla w(x)| \leq|Q(x)|_{o p}^{\frac{1}{2}}|\nabla w(x)| \leq c v(x)^{\frac{1}{2}}|\nabla w(x)|
$$

hence,

$$
\|\nabla w(x)\|_{L_{Q}^{2}(E)}^{2} \leq\|\nabla w\|_{\infty}^{2} v(E)=0
$$

which proves $(1)$.

Let $\mathbf{u} \in Q H_{0}^{1}(v ; \Omega)$; then there exists a sequence $\left\{w_{j}\right\}_{j} \subset \operatorname{Lip}_{0}(\Omega)$ such that $\nabla w_{j} \rightarrow \nabla u$ in $L_{Q}^{2}(\Omega)$. Then by the previous argument,

$$
\|\nabla u\|_{L_{Q}^{2}(E)}=\lim _{j \rightarrow \infty}\left\|\nabla w_{j}\right\|_{L_{Q}^{2}(E)}=0
$$

and so (2) holds.

Finally, (3) follows immediately from (1) and (2).

Our second result shows that non-negative truncations of functions in $Q H_{0}^{1}(v ; \Omega)$ are again in this space.

Lemma 2.14. Let $\mathbf{u}=(u, \nabla u) \in Q H_{0}^{1}(v ; \Omega)$ and fix $r>0$. If $S(r)=\{x \in \Omega$ : $u(x)>r\}$, then $\left((u-r)_{+}, \mathbb{1}_{S(r)} \nabla u\right) \in Q H_{0}^{1}(v ; \Omega)$.

Proof. By the definition of $Q H_{0}^{1}(v ; \Omega)$ there exists a sequence $\left\{u_{j}\right\}_{j}$ in $\operatorname{Lip}_{0}(\Omega)$ such that $u_{j} \rightarrow u$ in $L^{2}(v ; \Omega)$ and $\nabla u_{j} \rightarrow \nabla u$ in $L_{Q}^{2}(\Omega)$. If we pass to a subsequence, we may assume that $u_{j} \rightarrow u$ pointwise $v$-a.e. We will first prove that $\left(u_{j}-r\right)_{+} \rightarrow(u-r)_{+}$ in $L^{2}(v ; \Omega)$.

Define $f_{j}=\left|\left(u_{j}-r\right)_{+}-(u-r)_{+}\right|^{2}$; then $f_{j} \rightarrow 0 v$-a.e. We will show that $f_{j}$ converges to 0 in $L^{1}(v ; \Omega)$; this follows from the generalized dominated convergence 
theorem $\left[11\right.$, p. 59] if we show that there exist non-negative functions $g_{j}, g \in L^{1}(v ; \Omega)$ such that $f_{j} \leq g_{j}$ and $\left\|g_{j}\right\|_{L^{1}(v ; \Omega)} \rightarrow\|g\|_{L^{1}(v ; \Omega)}$ as $j \rightarrow \infty$. But we have that

$$
f_{j} \leq 2\left(u_{j}-r\right)_{+}^{2}+2(u-r)_{+}^{2} \leq 4\left(\left|u_{j}\right|^{2}+r^{2}\right)+4\left(|u|^{2}+r^{2}\right)=g_{j} .
$$

Moreover, $g_{j}$ converges pointwise a.e. to $g=8\left(|u|^{2}+r^{2}\right)$, and $g, g_{j} \in L^{1}(v ; \Omega)$ since $v(\Omega)<\infty$. Finally, since $u_{j} \rightarrow u$ in $L^{2}(v ; \Omega)$ we get that $\left\|g_{j}\right\|_{L^{1}(v ; \Omega)} \rightarrow\|g\|_{L^{1}(v ; \Omega)}$.

Now define $S_{j}=\left\{x \in \Omega: u_{j}(x)>r\right\}$; then $\mathbb{1}_{S_{j}} \rightarrow \mathbb{1}_{S}$ v-a.e. Moreover, we have that $\nabla\left(u_{j}-r\right)_{+}=\nabla u_{j} \mathbb{1}_{S_{j}}$ a.e. $[12$, Lemma 7.6] and so v-a.e. By passing to another subsequence, we assume that $\nabla u_{j} \mathbb{1}_{S_{j}} \rightarrow \nabla u \mathbb{1}_{S}$ pointwise $v$-a.e. We claim that they converge in $L_{Q}^{2}(\Omega)$ as well. If this is the case, then we have shown that $\left(\left(u_{j}-r\right)_{+}, \nabla\left(u_{j}-r\right)_{+}\right)$is Cauchy in $Q H_{0}^{1}(v ; \Omega)$, and the desired conclusion follows at once.

To prove $L_{Q}^{2}(\Omega)$ convergence, note that

$$
\left\|\nabla u_{j} \mathbb{1}_{S_{j}}-\nabla u \mathbb{1}_{S}\right\|_{L_{Q}^{2}(\Omega)} \leq\left\|\nabla u_{j} \mathbb{1}_{S_{j}}-\nabla u \mathbb{1}_{S_{j}}\right\|_{L_{Q}^{2}(\Omega)}+\left\|\sqrt{Q} \nabla u\left(\mathbb{1}_{S_{j}}-\mathbb{1}_{S}\right)\right\|_{L^{2}(\Omega)} .
$$

The first term on the right-hand side is less than $\left\|\nabla u_{j}-\nabla u\right\|_{L_{Q}^{2}(\Omega)}$ which goes to 0 as $j \rightarrow \infty$. To estimate the second term, let $E$ be the set of $x \in \Omega$ where $\mathbb{1}_{S_{j}}(x)$ does not converge to $\mathbb{1}_{S}(x)$. Then $v(E)=0$, and so by Lemma 2.13,

$$
\int_{E}\left|\sqrt{Q} \nabla u\left(\mathbb{1}_{S_{j}}-\mathbb{1}_{S}\right)\right|^{2} d x \leq \int_{E}|\sqrt{Q} \nabla u|^{2} d x=0 .
$$

Since $\left|\sqrt{Q} \nabla u\left(\mathbb{1}_{S_{j}}-\mathbb{1}_{S}\right)\right| \leq|\sqrt{Q} \nabla u| \in L^{2}(\Omega)$, by the dominated convergence theorem we have that as $j \rightarrow 0$,

$$
\left\|\sqrt{Q} \nabla u\left(\mathbb{1}_{S_{j}}-\mathbb{1}_{S}\right)\right\|_{L^{2}(\Omega)}=\left\|\sqrt{Q} \nabla u\left(\mathbb{1}_{S_{j}}-\mathbb{1}_{S}\right)\right\|_{L^{2}(\Omega \backslash E)} \rightarrow 0 .
$$

Our next lemma proves the existence of an approximating sequence of Lipschitz functions with some additional useful properties.

Lemma 2.15. Let $\mathbf{u}=(u, \nabla u) \in Q H_{0}^{1}(v ; \Omega)$ with $u \in L^{\infty}(v ; \Omega)$ and $u \geq 0$ v-a.e. Then there exists a sequence $\left\{u_{j}\right\}_{j} \in \operatorname{Lip}_{0}(\Omega)$ such that:

(1) $0 \leq u_{j}(x) \leq\|u\|_{L^{\infty}(v ; \Omega)}+1$ in $\Omega$;

(2) $u_{j} \rightarrow u$ v-a.e. and also in $L^{2}(v ; \Omega)$;

(3) $\nabla u_{j} \rightarrow \nabla u$ in $L_{Q}^{2}(\Omega)$ and $\left|\sqrt{Q} \nabla u_{j}\right| \rightarrow|\sqrt{Q} \nabla u|$ pointwise a.e.;

(4) $\left\|\nabla u_{j}\right\|_{L_{Q}^{2}(\Omega)} \leq\|\nabla u\|_{L_{Q}^{2}(\Omega)}+1$ for each $j \in \mathbb{N}$.

Proof. By the definition of $Q H_{0}^{1}(v ; \Omega)$ and by passing twice to a subsequence, there exists a sequence $\left\{z_{j}\right\}_{j} \subset \operatorname{Lip}_{0}(\Omega)$ such that:

$\left(1^{\prime}\right) z_{j} \rightarrow u$ both $v$-a.e. and also in $L^{2}(v ; \Omega)$

$\left(2^{\prime}\right) \nabla z_{j} \rightarrow \nabla u$ in $L_{Q}^{2}(\Omega)$ and $\left|\sqrt{Q} \nabla z_{j}\right| \rightarrow|\sqrt{Q} \nabla u|$ a.e.; 
$\left(3^{\prime}\right)\left\|\nabla z_{j}\right\|_{L_{Q}^{2}(\Omega)} \leq\|\nabla u\|_{L_{Q}^{2}(\Omega)}+1$.

Now let $w_{j}=\left|z_{j}\right|$. Since $u$ is non-negative $v$-a.e. in $\Omega$, by the triangle inequality we have that

$$
\left|w_{j}-u\right|=|| z_{j}|-| u|| \leq\left|z_{j}-u\right|
$$

$v$-a.e. Therefore, we have that $w_{j}$ converges to $u$ both in $L^{2}(v ; \Omega)$ and pointwise $v$-a.e.

By the Rademacher-Stepanov theorem [9], $\nabla w_{j}(x)=\operatorname{sgn}\left(z_{j}(x)\right) \nabla z_{j}(x)$ a.e. Hence, $\left|\sqrt{Q} \nabla w_{j}(x)\right|=\left|\sqrt{Q} \nabla z_{j}(x)\right|$ a.e. and so $\left\|\nabla w_{j}\right\|_{L_{Q}^{2}(\Omega)} \rightarrow\|\nabla u\|_{L_{Q}^{2}(\Omega)}$ as $j \rightarrow \infty$. Thus $w_{j} \geq 0$ a.e. and properties $\left(1^{\prime}\right)-\left(3^{\prime}\right)$ above hold with $z_{j}$ replaced by $w_{j}$.

We now define the sequence of $\operatorname{Lip}_{0}(\Omega)$ functions $\left\{u_{j}\right\}_{j}$. Set $M=\|u\|_{L^{\infty}(v ; \Omega)}+1$ and let $\phi:[0, \infty) \rightarrow[0, \infty)$ be such that $\phi \in C^{\infty}, \phi$ is increasing, $\phi(x)=x$ if $0 \leq x \leq M-\frac{1}{2}, \phi(x)=M$ if $x \geq M+1$, and $\phi^{\prime}(x) \leq 1$. Define the $u_{j}$ by $u_{j}(x)=\phi\left(w_{j}(x)\right)$. Then $\phi_{j} \in \operatorname{Lip}_{0}(\Omega)$; moreover, $\nabla u_{j}(x)=\phi^{\prime}\left(w_{j}(x)\right) \nabla w_{j}(x)$ a.e. and so

$$
\left|\sqrt{Q(x)} \nabla u_{j}(x)\right| \leq\left|\sqrt{Q(x)} \nabla w_{j}(x)\right|
$$

We claim that $\left\{u_{j}\right\}_{j}$ satisfies properties (1)-(4) above. By the definition of $\phi$, $0 \leq u_{j} \leq M$, so property (1) holds. Property (4) follow immediately from (2.16) and property $\left(3^{\prime}\right)$ for the $w_{j}$.

It remains to prove properties (2) and (3). By the choice of $M, u(s) \leq M-1$ for $v$-a.e. $s \in \Omega$. We also have that $w_{j}(s) \rightarrow u(s) v$-a.e. Let $F$ be the set of all $s \in \Omega$ such that both of these hold. Then $v(\Omega \backslash F)=0$. Given $s \in F$ there exists $N>0$ such that if $j \geq N, w_{j}(s)<M-\frac{1}{2}$, and so $u_{j}(s)=w_{j}(s)$. Thus, $u_{j} \rightarrow u$ pointwise $v$-a.e. Since $u$ is bounded and $v(\Omega)<\infty$, by the dominated convergence theorem we also have that $u_{j} \rightarrow u$ in $L^{2}(v ; \Omega)$. This proves $(2)$.

To prove (3) define the set $F$ as above. For each $s \in F$, there exists $N>0$ such that for each $j \geq N$ there exists a ball $B_{j, s}$ where for $x \in B_{j, s}, w_{j}(x)<M-\frac{1}{2}$; hence, $\nabla u_{j}(s)=\nabla w_{j}(s)$ for $j \geq N$. Now let $G$ be the set of $s \in \Omega$ such that $\left|\sqrt{Q(s)} \nabla w_{j}(s)\right| \rightarrow|\sqrt{Q(s)} \nabla u(s)|$; by $\left(2^{\prime}\right),|\Omega \backslash G|=0$. Since $v d x$ is an absolutely continuous measure, $v(\Omega \backslash G)=0$. Let $H=F \cap G$. Then on $H$ we have that $\left|\sqrt{Q} \nabla u_{j}\right| \rightarrow|\sqrt{Q} \nabla u|$ pointwise. But $v(\Omega \backslash H)=0$ so by Lemma 2.13 we have that

$$
\left\|\nabla u_{j}\right\|_{L_{Q}^{2}(\Omega \backslash H)}=0=\|\nabla u\|_{L_{Q}^{2}(\Omega \backslash H)} .
$$

This implies that $\left|\sqrt{Q} \nabla u_{j}\right|=0=|\sqrt{Q} \nabla u|$ almost everywhere on $\Omega \backslash H$. Therefore, we have that $\left|\sqrt{Q} \nabla u_{j}\right| \rightarrow|\sqrt{Q} \nabla u|$ pointwise a.e.

Finally, to prove that $\nabla u_{j} \rightarrow \nabla u$ in $L_{Q}^{2}(\Omega)$ we use the generalized dominated convergence theorem as in the proof of Lemma 2.14. Let $f_{j}=\left|\sqrt{Q}\left(\nabla u_{j}-\nabla u\right)\right|^{2}$; then $f_{j} \rightarrow 0$ a.e. Further, by (2.16)

$$
f_{j} \leq 2\left|\sqrt{Q} \nabla u_{j}\right|^{2}+2|\sqrt{Q} \nabla u|^{2} \leq 2\left|\sqrt{Q} \nabla w_{j}\right|^{2}+2|\sqrt{Q} \nabla u|^{2}=g_{j} .
$$


Again by $\left(2^{\prime}\right), g_{j} \rightarrow 4|\sqrt{Q} \nabla u|^{2}=g$ a.e., and since $\nabla w_{j} \rightarrow \nabla u$ in $L_{Q}^{2}(\Omega), g_{j} \rightarrow g$ in $L^{1}(\Omega)$. Therefore, $f_{j} \rightarrow 0$ in $L^{1}(\Omega)$, which completes the proof of $(3)$.

The next two lemmas give the product rule and chain rule associated to pairs in $Q H_{0}^{1}(\Omega)$. The proofs are adapted from those of similar results in [19].

Lemma 2.17. Let $(u, \nabla u) \in Q H_{0}^{1}(v ; \Omega)$ and let $\psi \in \operatorname{Lip}_{0}(\Omega)$. Then we have that $(u \psi, \psi \nabla u+u \nabla \psi) \in Q H_{0}^{1}(v ; \Omega)$.

Proof. By the definition of $Q H_{0}^{1}(v ; \Omega)$ there exists a sequence $\left\{w_{j}\right\} \subset \operatorname{Lip}_{0}(\Omega)$ such that $w_{j} \rightarrow u$ in $L^{2}(v ; \Omega)$ and $\nabla w_{j} \rightarrow \nabla u$ in $L_{Q}^{2}(\Omega)$. But then we immediately have that

$$
\left\|w_{j} \psi-u \psi\right\|_{L^{2}(v ; \Omega)} \leq\|\psi\|_{\infty}\left\|w_{j}-u\right\|_{L^{2}(v ; \Omega)},
$$

and so $w_{j} \psi \rightarrow u \psi$ in $L^{2}(v ; \Omega)$.

Similarly, since $|Q|_{o p} \leq k v$ a.e., we have that

$$
\begin{array}{r}
\left\|\nabla\left(w_{j} \psi\right)-(u \nabla \psi+\psi \nabla u)\right\|_{L_{Q}^{2}(\Omega)} \leq\left\|\psi \nabla w_{j}-\psi \nabla u\right\|_{L_{Q}^{2}(\Omega)}+\left\|w_{j} \nabla \psi-u \nabla \psi\right\|_{L_{Q}^{2}(\Omega)} \\
\leq\|\psi\|_{\infty}\left\|\nabla w_{j}-\nabla u\right\|_{L_{Q}^{2}(\Omega)}+k\|\nabla \psi\|_{\infty}\left\|w_{j}-u\right\|_{L^{2}(v ; \Omega)} .
\end{array}
$$

Thus, $\nabla\left(w_{j} \psi\right) \rightarrow u \nabla \psi+\psi \nabla u$ in $L_{Q}^{2}(\Omega)$ and so $(u \psi, u \nabla \psi+\psi \nabla u) \in Q H_{0}^{1}(v ; \Omega)$.

Lemma 2.18. Let $(u, \nabla u) \in Q H_{0}^{1}(v ; \Omega)$ with $u \geq 0$ v-a.e. and $u \in L^{\infty}(v ; \Omega)$. Then, given any non-negative function $\varphi \in C^{1}(\mathbb{R})$ such that $\varphi(0)=0$, the pair $\left(\varphi(u), \varphi^{\prime}(u) \nabla u\right) \in Q H_{0}^{1}(v ; \Omega)$.

Proof. Let $\left\{u_{j}\right\}_{j} \subset \operatorname{Lip}_{0}(\Omega)$ be the sequence associated with $(u, \nabla u)$ given by Lemma 2.15. Since $u_{j}$ is Lipschitz with compact support in $\Omega$ and $\varphi(0)=0, \psi_{j}=\varphi\left(u_{j}\right) \in$ $\operatorname{Lip}_{0}(\Omega)$. Since $u_{j} \rightarrow u v$-a.e., the continuity of $\varphi$ implies that $\psi_{j} \rightarrow \varphi(u)=\psi v$-a.e. By the fundamental theorem of calculus,

$$
|\varphi(t)|=\left|\int_{0}^{t} \varphi^{\prime}(s) d s\right| \leq\left\|\varphi^{\prime}\right\|_{L^{\infty}([0, M])}|t|=A_{0}|t|
$$

whenever $0 \leq t \leq M=\|u\|_{L^{\infty}(v ; \Omega)}+1$.

Since by assumption and property (1) of Lemma 2.15, $0 \leq u(x), u_{j}(x) \leq M$ for $v$-a.e. $x \in \Omega$, we have that $v$-a.e.,

$$
\left|\psi_{j}-\psi\right|^{2} \leq 2\left(\left|\psi_{j}\right|^{2}+|\psi|^{2}\right) \leq 2 A_{0}^{2}\left(\left|u_{j}\right|^{2}+|u|^{2}\right)
$$

Since $\left|u_{j}\right|^{2}+|u|^{2} \rightarrow 2|u|^{2} v$-a.e. and in $L^{1}(v ; \Omega)$, by the generalized Lebesgue dominated convergence theorem we get that $\psi_{j} \rightarrow \psi$ in $L^{2}(v ; \Omega)$.

To show the convergence of the gradients, first note that $\sqrt{Q} \nabla \psi_{j}=\varphi^{\prime}\left(u_{j}\right) \sqrt{Q} \nabla u_{j}$ a.e. in $\Omega$ and so by the continuity of $\varphi^{\prime}$ and property (3) in Lemma 2.15 we get that $\sqrt{Q} \nabla \psi_{j} \rightarrow \varphi^{\prime}(u) \sqrt{Q} \nabla u$ a.e. Moreover, 


$$
\begin{aligned}
\left|\sqrt{Q} \nabla\left(\psi_{j}\right)-\varphi^{\prime}(u) \sqrt{Q} \nabla u\right|^{2} \leq 2\left|\varphi^{\prime}\left(u_{j}\right) \sqrt{Q} \nabla u_{j}\right|^{2} & +2\left|\varphi^{\prime}(u) \sqrt{Q} \nabla u\right|^{2} \\
& \leq 2 A_{0}^{2}\left(\left|\sqrt{Q} \nabla u_{j}\right|^{2}+|\sqrt{Q} \nabla u|^{2}\right) .
\end{aligned}
$$

The right-hand term converges to $4 A_{0}^{2}|\sqrt{Q} \nabla u|^{2}$ both pointwise a.e. and in $L^{1}(\Omega)$. Therefore, we can again apply the generalized dominated convergence theorem to get that $\nabla \psi_{j} \rightarrow \varphi^{\prime}(u) \nabla u$ in $L_{Q}^{2}(\Omega)$. We conclude that $\left(\varphi(u), \varphi^{\prime}(u) \nabla u\right) \in Q H_{0}^{1}(v ; \Omega)$.

Exponential results. In this section we give two results which are needed to prove Theorem 1.11. The first gives a solution to an auxiliary Dirichlet problem and is an application of the previous two lemmas.

Lemma 2.19. Fix $\alpha>0$. If $(u, \nabla u) \in Q H_{0}^{1}(\Omega)$ is a non-negative bounded weak subsolution of the Dirichlet problem

$$
\left\{\begin{aligned}
-\operatorname{Div}(Q \nabla u) & =\text { fv } \\
u & =0 \quad \text { for } x \in \Omega, \\
& \text { for } x \in \partial \Omega,
\end{aligned}\right.
$$

then $(w, \nabla w)=\left(e^{\alpha u}-1, \alpha e^{\alpha u} \nabla u\right) \in Q H_{0}^{1}(v ; \Omega)$ is a non-negative weak subsolution of the Dirichlet problem

$$
\left\{\begin{aligned}
-\operatorname{Div}(Q \nabla w) & =\alpha f(w+1) v & & \text { for } x \in \Omega, \\
w & =0 & & \text { for } x \in \partial \Omega .
\end{aligned}\right.
$$

Proof. Fix a non-negative $\psi \in \operatorname{Lip}_{0}(\Omega)$. By our assumptions on $(u, \nabla u)$ and by Lemmas 2.17 and 2.18 we have that that both $(w, \nabla w)=\left(e^{\alpha u}-1, \alpha e^{\alpha u} \nabla u\right)$ and $(\psi(w+1),(w+1) \nabla \psi+\psi \nabla w)$ are in $Q H_{0}^{1}(\Omega)$. Since $\nabla w=\alpha(w+1) \nabla u$ and $(u, \nabla u)$ is a non-negative weak subsolution of $(2.20)$, we have that

$$
\begin{aligned}
\int_{\Omega} f(w+1) \psi v d x & \geq \int_{\Omega} \nabla(\psi(w+1)) Q \nabla u d x \\
& =\int_{\Omega}(w+1) \nabla \psi Q \nabla u d x+\int_{\Omega} \psi \nabla(w+1) Q \nabla w d x \\
& =\frac{1}{\alpha} \int_{\Omega} \nabla \psi Q \nabla w d x+\int_{\Omega} \psi \nabla w Q \nabla w d x \\
& \geq \frac{1}{\alpha} \int_{\Omega} \nabla \psi Q \nabla w d x
\end{aligned}
$$

Since $\psi \in \operatorname{Lip}_{0}(\Omega)$ is arbitrary, we conclude that $w$ is a non-negative weak subsolution of (4.7).

Our second result gives the exponential integrability of bounded solutions to (1.1). A version of this result is proved in [26, Lemma B] for uniformly elliptic operators; a qualitative version appeared previously in [4, Example 4]. Here we adapt the proof from [26] to our more general setting. 
Lemma 2.22. Suppose Hypothesis 1.4 holds. Let $f \in L^{\sigma^{\prime}}(v ; \Omega)$ satisfy $\|f\|_{\sigma^{\prime} ; v} \leq 1$, and let $(u, \nabla u) \in Q H_{0}^{1}(\Omega)$ be a bounded, non-negative weak subsolution of (1.1). Then, for every $\gamma \in\left(0, \frac{4}{C_{0}^{2}}\right)$, with $C_{0}$ as in $(1.5)$, there $M=M\left(\gamma, C_{0}, v(\Omega)\right)$ such that

$$
\int_{\Omega} e^{\gamma u(x)} v(x) d x \leq M .
$$

Proof. Let $f$ and $(u, \nabla u)$ be as in the hypotheses. Define $\varphi=e^{\gamma u}-1$ and $\psi=e^{\frac{\gamma u}{2}}-1$ with $\gamma>0$ to be chosen below. Since $u$ is bounded, by Lemma 2.18 we have that

$$
(\varphi, \nabla \varphi)=\left(e^{\gamma u}-1, \gamma e^{\gamma u} \nabla u\right), \quad(\psi, \nabla \psi)=\left(e^{\frac{\gamma u}{2}}-1, \frac{\gamma}{2} e^{\frac{\gamma u}{2}} \nabla u\right)
$$

are in $Q H_{0}^{1}(\Omega)$. Further, we immediately have the identities $\varphi=\psi^{2}+2 \psi, \nabla \psi=$ $\frac{\gamma}{2} e^{\frac{\gamma u}{2}} \nabla u$, and $\nabla \varphi=2 e^{\frac{\gamma u}{2}} \nabla \psi$. If we apply the Sobolev inequality (2.10) and use $\varphi$ as a test function in Definition 2.11 we can estimate as follows:

$$
\begin{aligned}
\|\psi\|_{L^{2 \sigma}(v ; \Omega)}^{2} & \leq C_{0}^{2} \int_{\Omega}|\sqrt{Q(x)} \nabla \psi(x)|^{2} d x \\
& =\frac{C_{0}^{2} \gamma}{4} \int_{\Omega} \nabla \varphi(x) \cdot Q(x) \nabla u(x) d x \\
& \leq \frac{C_{0}^{2} \gamma}{4} \int_{\Omega} f(x) \varphi(x) v(x) d x \\
& =\frac{C_{0}^{2} \gamma}{4}\left(\int_{\Omega} f(x) \psi(x)^{2} v(x) d x+2 \int_{\Omega} f(x) \psi(x) v(x) d x\right) .
\end{aligned}
$$

If we now apply Hölder's inequality with exponents $\sigma$ and $2 \sigma$, and then 2 , we get

$$
\begin{aligned}
& =\frac{C_{0}^{2} \gamma}{4}\left(\|f\|_{L^{\sigma^{\prime}(v ; \Omega)}}\left\|\psi^{2}\right\|_{L^{\sigma}(v ; \Omega)}+2\|f\|_{L^{\sigma^{\prime}}(v ; \Omega)}\|\psi\|_{L^{\sigma}(v ; \Omega)}\right) \\
& \leq \frac{C_{0}^{2} \gamma}{4}\left(\|\psi\|_{L^{2 \sigma}(v ; \Omega)}^{2}+2\|\psi\|_{L^{2 \sigma}(v ; \Omega)} v(\Omega)^{\frac{1}{2 \sigma}}\right) .
\end{aligned}
$$

If we now fix $\gamma \in\left(0, \frac{4}{C_{0}^{2}}\right)$, then we can re-arrange terms to get

$$
\|\psi\|_{L^{2 \sigma}(v ; \Omega)} \leq \frac{C_{0}^{2} \gamma}{2\left(1-\frac{C_{0}^{2} \gamma}{4}\right)} v(\Omega)^{\frac{1}{2 \sigma}} .
$$

Therefore, again by Hölder's inequality and by (2.24) applied twice, we have that

$$
\begin{aligned}
\int_{\Omega} e^{\gamma u(x)} v(x) d x & =\int_{\Omega}\left(\psi(x)^{2}+2 \psi(x)\right) v(x) d x+v(\Omega) \\
& \leq\|\psi\|_{L^{2 \sigma}(v ; \Omega)}^{2} v(\Omega)^{\frac{1}{\sigma}}+2\|\psi\|_{L^{2 \sigma}(v ; \Omega)} v(\Omega)^{\frac{1}{(2 \sigma)^{\prime}}}+v(\Omega) \\
& \leq C\left(\gamma, C_{0}\right) v(\Omega)
\end{aligned}
$$




$$
=M\left(\gamma, C_{0}, v(\Omega)\right)
$$

\section{Proof of Theorem 1.7}

Fix $f \in L^{A}(v ; \Omega)$ and let $\mathbf{u}=(u, \nabla u) \in Q H_{0}^{1}(\Omega)$ be a non-negative weak subsolution of (1.1). We may assume without loss of generality that $\|f\|_{L^{A}(v ; \Omega)}>0$ (equivalently, that $f$ is non-zero on a set $E \subset \Omega$ with $v(E)>0$ ); otherwise, a standard argument shows that $u=0 v$-almost everywhere. (Cf. (3.8) below.) By Lemma 2.6, $f \in L^{\sigma^{\prime}}(v ; \Omega)$.

For each $r>0$ define $\varphi_{r}=(u-r)_{+}$and let $S(r)=\{x \in \Omega: u(x)>r\}$. Then by Lemma 2.14, $\left(\varphi_{r}, \nabla \varphi_{r}\right)=\left((u-r)_{+}, \mathbb{1}_{S(r)} \nabla u\right) \in Q H_{0}^{1}(v ; \Omega)$. We now estimate as follows: by the Sobolev inequality (2.10), the definition of a weak subsolution with $\varphi_{r}$ as the test function, and Hölder's inequality, we have that

$$
\begin{aligned}
& \left\|\varphi_{r}\right\|_{L^{2 \sigma}(v ; \Omega)}^{2} \leq C_{0}^{2} \int_{S(r)}\left|\sqrt{Q} \nabla \varphi_{r}\right|^{2} d x=C_{0}^{2} \int_{S(r)} \nabla \varphi_{r} \cdot Q \nabla \varphi_{r} d x \\
& \quad=C_{0}^{2} \int_{S(r)} \nabla \varphi_{r} \cdot Q \nabla u d x \leq C_{0}^{2} \int_{S(r)} f \varphi_{r} v d x \leq C_{0}^{2}\|f\|_{L^{(2 \sigma)^{\prime}(v ; S(r))}}\left\|\varphi_{r}\right\|_{L^{2 \sigma}(v ; \Omega)}
\end{aligned}
$$

since $\nabla u=\nabla \varphi_{r}$ on $S(r)$. If we divide through by $\left\|\varphi_{r}\right\|_{L^{2 \sigma}(v ; \Omega)}$, we get

$$
\left\|\varphi_{r}\right\|_{L^{2 \sigma}(v ; \Omega)} \leq C\|f\|_{L^{(2 \sigma)^{\prime}(v ; S(r))}} .
$$

In order to estimate the norm of the right-hand side, recall that since $\sigma>1$, $(2 \sigma)^{\prime}<\sigma^{\prime}$, we can define the Young function

$$
B(t)=t^{\frac{\sigma^{\prime}}{(2 \sigma)^{\prime}}} \log (e+t)^{q} .
$$

It is immediate that $B_{\sigma}(t)=B\left(t^{(2 \sigma)^{\prime}}\right) \preceq A(t)$ and so by Lemma 2.2, a change of variables in the Luxemburg norm, and Lemmas 2.1 and 2.7 we get

$$
\begin{aligned}
\|f\|_{L^{(2 \sigma)^{\prime}(v ; S(r))}}^{(2 \sigma)^{\prime}} & =\int_{\Omega}|f|^{(2 \sigma)^{\prime}} \mathbb{1}_{S(r)} v d x \\
& \leq 2\left\|f^{(2 \sigma)^{\prime}}\right\|_{L^{B}(v ; \Omega)}\left\|\mathbb{1}_{S(r)}\right\|_{L^{\bar{B}}(v ; \Omega)} \\
& =2\|f\|_{L^{B \sigma}(v ; \Omega)}^{(2 \sigma)^{\prime}}\left\|\mathbb{1}_{S(r)}\right\|_{L^{\bar{B}}(v ; \Omega)} \\
& \leq C\|f\|_{L^{A}(v ; \Omega)}^{(2 \sigma)^{\prime}} \frac{v(S(r))^{\frac{1}{2 \sigma-1}}}{\log \left(e+(v(S(r)))^{-1}\right)^{q\left(\frac{(2 \sigma)^{\prime}}{\sigma^{\prime}}\right)}},
\end{aligned}
$$

where $C=C(\sigma, q, v(\Omega))$ is independent of $f, \varphi$, and $\mathbf{u}$. 
We now turn to our iteration argument. For all $s>r, S(s) \subset S(r)$ and, for $x \in S(s), \varphi_{r}(x)>s-r>0$. Hence, if we combine the above two inequalities, we get

$$
v(S(s))^{\frac{1}{2 \sigma}}(s-r) \leq\left\|\varphi_{r} \mathbb{1}_{S(s)}\right\| L^{2 \sigma}(v ; \Omega) \leq C\|f\|_{L^{A}(v ; \Omega)} \frac{v(S(r))^{\frac{1}{2 \sigma}}}{\log \left(e+(v(S(r)))^{-1}\right)^{\frac{q}{\sigma^{\sigma}}}} .
$$

Define $r_{0}=\tau_{0}\|f\|_{L^{A}(v ; \Omega)}$ with $\tau_{0}$ to be chosen below. Our goal is to find $\tau_{0}$ sufficiently large so that $v\left(S\left(r_{0}\right)\right)=0$, as this immediately implies that

$$
\|u\|_{L^{\infty}(v ; \Omega)} \leq \tau_{0}\|f\|_{L^{A}(v ; \Omega)},
$$

which is what we want to prove. To do this, we will use an iteration argument based on De Giorgi iteration. For each $k \in \mathbb{N}$ set

$$
C_{k}=r_{0}\left(1-(k+1)^{-\epsilon}\right)
$$

where $\epsilon>0$ will be chosen below, and let $C_{0}=C_{1} / 2$. The sequence $\left\{C_{k}\right\}_{k=0}^{\infty}$ increases to $r_{0}$ and by an estimate using the mean-value theorem we have that for each $k \in \mathbb{N}$,

$$
C_{k+1}-C_{k} \geq \frac{\epsilon r_{0}}{(k+2)^{1+\epsilon}}
$$

If we set $s=C_{k+1}, r=C_{k}, \mu_{k}=v\left(S\left(C_{k}\right)\right)$ in inequality (3.2), we get

$$
\mu_{k+1} \leq\left[\frac{C(k+2)^{1+\epsilon}}{\epsilon \tau_{0}}\right]^{2 \sigma} \frac{\mu_{k}}{\log \left(e+\mu_{k}^{-1}\right)^{\frac{2 q \sigma}{\sigma^{\prime}}}}
$$

for each $k \in \mathbb{N}$. By the dominated convergence theorem $\mu_{k}$ converges to $v\left(S\left(r_{0}\right)\right)$, so to complete the proof we need to prove that $\mu_{k} \rightarrow 0$.

Let $m_{k}=\log \left(\mu_{k}^{-1}\right)$. We will show that $m_{k} \rightarrow \infty$ as $k \rightarrow \infty$, which is equivalent to the desired limit. To do so, we will show that we can choose $\epsilon$ and $\tau_{0}$ such that $m_{0} \geq 2$ and

$$
m_{k} \geq m_{0}+k
$$

for all $k \in \mathbb{N} \cup\{0\}$.

Fix $\epsilon=\frac{q}{\sigma^{\prime}}-1>0$. Since $2 \sigma(1+\epsilon)=\frac{2 \sigma q}{\sigma^{\prime}}$, if we take logarithms and re-arrange terms, inequality (3.5) becomes, for $k \in \mathbb{N}$,

$$
m_{k+1} \geq 2 \sigma \log \left(\frac{\epsilon \tau_{0}}{C}\right)+\frac{2 \sigma q}{\sigma^{\prime}} \log \left(\frac{m_{k}}{k+2}\right)+m_{k} .
$$

The first step is to fix $m_{0}$ by an appropriate choice of $\tau_{0}>0$. If we argue as we did to prove (3.1) using $u$ as the test function in the definition of a weak subsolution, we get

$$
\|u\|_{L^{2 \sigma}(v ; \Omega)} \leq C\|f\|_{L^{(2 \sigma)^{\prime}(v ; \Omega)}}
$$


If we estimate the right-hand side using Hölder's inequality and Lemma 2.6, we get

$$
\|f\|_{L^{(2 \sigma)^{\prime}(v ; \Omega)}} \leq C v(\Omega)^{\frac{1}{2 \sigma}}\|f\|_{L^{A}(v ; \Omega)},
$$

where the constant $C$ is independent of $f$ and $\mathbf{u}$. For each $x \in S\left(C_{0}\right)$ we have that $2 u(x) / C_{1}>1$, so by Hölder's inequality and the above two estimates,

$$
\begin{aligned}
& v\left(S\left(C_{0}\right)\right) \leq \frac{2}{C_{1}} \int_{S\left(C_{0}\right)} u v d x \leq \frac{2}{C_{1}}\|u\|_{L^{2 \sigma}(v ; \Omega)} v\left(S\left(C_{0}\right)\right)^{\frac{1}{(2 \sigma)^{\prime}}} \\
& \quad \leq \frac{2 C}{C_{1}} v(\Omega)^{\frac{1}{2 \sigma}}\|f\|_{L^{A}(v ; \Omega)} v\left(S\left(C_{0}\right)\right)^{\frac{1}{(2 \sigma)^{\prime}}} \leq \frac{2 C v(\Omega)^{\frac{1}{2 \sigma}}}{\tau_{0}\left(1-2^{-\epsilon}\right)} v\left(S\left(C_{0}\right)\right)^{\frac{1}{(2 \sigma)^{\prime}}} .
\end{aligned}
$$

If we re-arrange terms, we get

$$
v\left(S\left(C_{0}\right)\right) \leq\left(\frac{C}{\tau_{0}\left(1-2^{-\epsilon}\right)}\right)^{2 \sigma},
$$

where again the constant $C$ is independent of $f$ and $\mathbf{u}$. Now choose $\tau_{0}>0$ so that

$$
\mu_{0}=v\left(S\left(C_{0}\right)\right)<e^{-2}, \text { and } \tau_{0} \geq \max \left\{\frac{2^{\epsilon+1} e C}{2^{\epsilon}-1}, \frac{e C}{\epsilon}\right\},
$$

where $C$ is as in (3.2). Note that $\tau_{0}$ is independent of $\mathbf{u}$ and $f$, and the first inequality implies that $m_{0} \geq 2$.

It is clear that $m_{0} \geq m_{0}$ but for the sake of clarity we also show that $m_{1}>m_{0}+1$. Since $k=0$ we cannot use (3.7), but instead use (3.2) directly. If we set $s=C_{1}$ and $r=C_{0}$ we find

$$
\frac{C_{1}}{2} \mu_{1}^{\frac{1}{2 \sigma}} \leq C\|f\|_{L^{A}(v ; \Omega)} \frac{\mu_{0}^{\frac{1}{\ell(2 \sigma)}}}{\log \left(e+\mu_{0}^{-1}\right)^{\frac{q}{\sigma^{\prime}}}} .
$$

If we use the definition of $C_{1}$ and recall that $m_{j}=\log \left(\mu_{j}^{-1}\right)$, we get

$$
\begin{aligned}
m_{1} \geq 2 \sigma \log \left(\frac{\left(2^{\epsilon}-1\right) \tau_{0}}{2^{\epsilon+1} C}\right)+m_{0}+2 q(\sigma-1) \log \left(m_{0}\right) & \\
& \geq \log \left(\frac{\left(2^{\epsilon}-1\right) \tau_{0}}{2^{\epsilon+1} e C}\right)+m_{0}+1 \geq m_{0}+1 ;
\end{aligned}
$$

the second inequality follows since $m_{0} \geq 1$, and the third by our choice of $\tau_{0}$.

Now suppose that $m_{j} \geq m_{0}+j$ for some $j \in \mathbb{N}$. Since $m_{0} \geq 2$, (3.7) and (3.9) together show that

$$
\begin{aligned}
m_{j+1} \geq 2 \sigma \log \left(\frac{\epsilon \tau_{0}}{C}\right)+\frac{2 \sigma q}{\sigma^{\prime}} \log \left(\frac{2+j}{2+j}\right) & +m_{0}+j \\
& \geq \log \left(\frac{\epsilon \tau_{0}}{e C}\right)+m_{0}+j+1 \geq m_{0}+j+1 .
\end{aligned}
$$


Hence, by induction we have that inequality (3.6) holds for all $k$, and this completes our proof.

\section{Proof of Theorem 1.11}

Our proof requires one technical lemma.

Lemma 4.1. Given $\sigma>1$, there exist constants $b \in(\sigma, 2 \sigma), \bar{b} \in\left((2 \sigma)^{\prime}, \sigma^{\prime}\right)$, and $p>1$ such that

$$
\frac{1}{b}+\frac{1}{\bar{b}}+\frac{1}{p}=1
$$

and

$$
\Gamma=\frac{2 \sigma}{\bar{b}}\left(\frac{\sigma^{\prime}-\bar{b}}{\sigma^{\prime}}+\frac{2 \sigma-b}{2 \sigma}\right)=1 .
$$

Proof. We will first show that we can choose $b$ and $\bar{b}$ so that (4.3) holds, and then show that we can refine our choice so that (4.2) holds as well.

Set $b=2 \sigma(1-\beta)$ and $\bar{b}=(1+\beta)(2 \sigma)^{\prime}$, where $0<\beta<\min \left(\frac{1}{2}, \frac{\sigma^{\prime}-(2 \sigma)^{\prime}}{(2 \sigma)^{\prime}}\right)$ will be determined below. With this restriction on $\beta$ it is immediate that $b$ and $\bar{b}$ lie in the specified intervals. Moreover, if we insert these values into the definition of $\Gamma$, we get

$$
\begin{aligned}
\Gamma=\frac{2 \sigma}{(1+\beta)(2 \sigma)^{\prime}} & \left(\frac{\sigma^{\prime}-(1+\beta)(2 \sigma)^{\prime}}{\sigma^{\prime}}+\frac{2 \sigma-2 \sigma(1-\beta)}{2 \sigma}\right) \\
& =\frac{2 \sigma}{(1+\beta)(2 \sigma)^{\prime}}\left((1+\beta)\left(1-\frac{(2 \sigma)^{\prime}}{\sigma^{\prime}}\right)\right)=2 \sigma\left(\frac{1}{(2 \sigma)^{\prime}}-\frac{1}{\sigma^{\prime}}\right)=1 .
\end{aligned}
$$

This gives (4.3).

To show that we can choose $p>1$ and $\beta$ so that (4.2) holds, note that

$$
\frac{1}{b}+\frac{1}{\bar{b}}=\frac{1}{2 \sigma(1-\beta)}+\frac{2 \sigma-1}{2 \sigma(1+\beta)}=\frac{1+\beta+2 \sigma-1-2 \beta \sigma+\beta}{2 \sigma\left(1-\beta^{2}\right)}=\frac{\sigma-\beta \sigma+\beta}{\sigma\left(1-\beta^{2}\right)} .
$$

Thus, $\frac{1}{b}+\frac{1}{b}<1$ exactly when $0<\beta<\frac{1}{\sigma^{\prime}}$. Hence, if we choose $\beta$ sufficiently small we can find $p>1$ such that (4.2) holds.

Remark 4.4. In the proof of Lemma 4.1, the range of possible values for $\beta$ shrinks as the dimension increases. In the classical case, $\sigma^{\prime}=\frac{n}{2}$, and this value is generally a lower bound on $\sigma^{\prime}$ in the more degenerate settings.

Proof of Theorem 1.11. Let $\mathbf{u}=(u, \nabla u) \in Q H_{0}^{1}(v ; \Omega)$ be a non-negative weak subsolution of (1.1). By the homogeneity of equation (1.1) and inequality (1.12), to prove this result it will suffice to assume that $\|f\|_{L^{\sigma^{\prime}(v ; \Omega)}}=1$ and prove that

$$
\|u\|_{L^{\infty}(v ; \Omega)} \leq C\left[1+\log \left(1+\|f\|_{L^{A}(v ; \Omega)}\right)\right] .
$$


To prove (4.5) we will apply an iteration argument very similar to that in the proof of Theorem 1.7, but to the solution of an auxiliary equation we which now define. Given that $\|f\|_{L^{\sigma^{\prime}(v ; \Omega)}}=1$, and since by Theorem $1.7 u$ is bounded in $\Omega$, we can apply Lemma 2.22 and fix $\gamma \in\left(0, \frac{4}{C_{0}^{2}}\right)$ such that

$$
\int_{\Omega} e^{\gamma u(x)} v(x) d x \leq M\left(\gamma, C_{0}, v(\Omega)\right)=M .
$$

Define $h=e^{\gamma u / p}$ (where $p>1$ will be determined below) and let $w=h-1$. By Lemma 2.19, $\left(w, \frac{\gamma}{p} h \nabla u\right) \in Q H_{0}^{1}(v ; \Omega)$ is a non-negative weak subsolution of

$$
\left\{\begin{aligned}
-\operatorname{Div}(Q \nabla w) & =\alpha f h v & & \text { for } x \in \Omega \\
w & =0 & & \text { for } x \in \partial \Omega .
\end{aligned}\right.
$$

For each $r>0$, let $\varphi_{r}=(w-r)_{+}$and $S(r)=\{x \in \Omega: w(x)>r\}$. By Lemma 2.14, $\left(\varphi_{r}, \nabla \varphi_{r}\right) \in Q H_{0}^{1}(v ; \Omega)$. By Lemma 4.1, there exist $\bar{b} \in\left((2 \sigma)^{\prime}, \sigma^{\prime}\right), b \in(\sigma, 2 \sigma)$, and $p>1$ such that (4.2) holds. We can now argue as we did in the proof of Theorem 1.7 with $\varphi_{r}$ as a test function, and then apply Hölder's inequality twice to get

$$
\begin{aligned}
\left\|\varphi_{r}\right\|_{L^{2 \sigma}(v ; \Omega)}^{2} & \leq C \int_{S(r)} \nabla \varphi_{r} Q \nabla \varphi_{r} d x \\
& =C \int_{S(r)} \nabla \varphi_{r} Q \nabla w d x \\
& \leq C \int_{S(r)} f \varphi_{r} h v d x \\
& \leq C\left\|f \mathbb{1}_{S(r)}\right\|_{L^{\bar{b}(v ; \Omega)}}\left\|\varphi_{r}\right\|_{L^{b}(v ; \Omega)}\|h\|_{L^{p}(v ; \Omega)} \\
& \leq C\left\|f \mathbb{1}_{S(r)}\right\|_{L^{\bar{b}}(v ; \Omega)}\left\|\varphi_{r}\right\|_{L^{2 \sigma}(v ; \Omega)} v(S(r))^{\frac{2 \sigma-b}{2 \sigma}} ;
\end{aligned}
$$

the last inequality follows since $b<2 \sigma$ and since by (4.6), $h \in L^{p}(v ; \Omega)$ with a constant independent of $\mathbf{u}$ and $f$.

Now define the Young function $B(t)=t^{\frac{\sigma^{\prime}}{b}} \log (e+t)^{q}$ and note that $B\left(|t|^{\bar{b}}\right) \preceq A(t)$. Therefore, arguing as before, by Lemma 2.7 and (4.8) we have that

$$
\left\|\varphi_{r}\right\|_{2 \sigma} \leq C\|f\|_{A} \frac{v(S(r))^{\frac{1}{b\left(\sigma^{\prime} / b\right)^{\prime}}+\frac{2 \sigma-b}{2 \sigma b}}}{\log \left(e+v\left(S(r)^{-1}\right)\right)^{\frac{q}{\sigma^{\prime}}}}=C\|f\|_{A} \frac{v(S(r))^{\frac{\sigma^{\prime}-\bar{b}}{b \sigma^{\prime}}+\frac{2 \sigma-b}{2 \sigma b}}}{\log \left(e+v(S(r))^{-1}\right)^{\frac{q}{\sigma^{\prime}}}}
$$

We can now argue as we did in the proof of Theorem 1.7 to get that for all $s>r$,

$$
v(S(s)) \leq\left(\frac{C\|f\|_{A}}{(s-r)}\right)^{2 \sigma} \frac{v(S(r))^{\frac{2 \sigma}{b}\left(\frac{\sigma^{\prime}-\bar{b}}{\sigma^{\prime}}+\frac{2 \sigma-b}{2 \sigma}\right)}}{\log \left(e+v(S(r))^{-1}\right)^{\frac{2 q \sigma}{\sigma^{\prime}}}}=\left(\frac{C\|f\|_{A}}{(s-r)}\right)^{2 \sigma} \frac{v(S(r))}{\log \left(e+v(S(r))^{-1}\right)^{\frac{2 q \sigma}{\sigma^{\prime}}}} ;
$$

the last inequality holds by (4.3). 
We continue the proof of Theorem 1.7 and define $\epsilon=\frac{q}{\sigma^{\prime}}-1>0, C_{k}, k \geq 0$, as in (3.3), and $m_{k}=-\log (v(S(C(k)))$ to again get the iteration inequality

$$
m_{k+1} \geq 2 \sigma \log \left(\frac{\epsilon \tau_{0}}{C}\right)+\frac{2 \sigma q}{\sigma^{\prime}} \log \left(\frac{m_{k}}{k+2}\right)+m_{k} .
$$

We will again prove that we can choose the parameter $\tau_{0}$ such that $m_{0}>1$ and for every $k \in \mathbb{N} \cup\{0\}$,

$$
m_{k} \geq m_{0}+k
$$

Assume for the moment that (4.10) holds. Then arguing as before we have that $\|w\|_{\infty} \leq \tau_{0}\|f\|_{A}$ : that is,

$$
e^{c\|u\|_{\infty}} \leq \tau_{0}\left(\|f\|_{A}+1\right),
$$

which in turn implies that (4.5) holds as desired.

Therefore, to complete the proof we need to show that (4.10) holds. The proof is almost identical to the proof of (3.6): the only difference is in the choice of $m_{0}$ which we will describe. We first estimate as we did for inequality (4.8):

$$
\begin{aligned}
& \|w\|_{L^{b}(v ; \Omega)}^{2} \leq\|w\|_{L^{2 \sigma}(v ; \Omega)}^{2} v(\Omega)^{\frac{2 \sigma-b}{\sigma}} \leq C \int_{\Omega} f w h v d x v(\Omega)^{\frac{2 \sigma-b}{\sigma}} \\
& \quad \leq C\|f\|_{L^{\bar{b}}(v ; \Omega)}\|w\|_{L^{b}(v ; \Omega)}\|h\|_{L^{p}(v ; \Omega)} v(\Omega)^{\frac{2 \sigma-b}{\sigma}} \leq C\|f\|_{L^{\bar{b}}(v ; \Omega)}\|w\|_{L^{b}(v ; \Omega)} v(\Omega)^{\frac{2 \sigma-b}{\sigma}},
\end{aligned}
$$

where the last inequality holds since $h \in L^{p}(v ; \Omega)$ with norm bounded by a constant. Furthermore, by Hölder's inequality and Lemma 2.6,

$$
\|f\|_{L^{\bar{b}}(v ; \Omega)}^{\bar{b}} \leq\|f\|_{L^{\sigma^{\prime}(v ; \Omega)}}^{\bar{b}} v(\Omega)^{\frac{1}{\left(\sigma^{\prime} / b\right)^{\prime}}} \leq\|f\|_{L^{A}(v ; \Omega)}^{\bar{b}} v(\Omega)^{\frac{1}{\left(\sigma^{\prime} / b\right)^{\prime}}} .
$$

Since $C_{0}=C_{1} / 2$, for every $x \in S\left(C_{0}\right)$ we have $\frac{2 w(x)}{C_{1}}>1$. Thus, combining the above inequalities, we get

$$
\begin{aligned}
v\left(S\left(C_{0}\right)\right) \leq & \frac{2}{C_{1}} \int_{S\left(C_{0}\right)} w v d x \leq \frac{2}{C_{1}}\|w\|_{L^{b}(v ; \Omega)} v\left(S\left(C_{0}\right)\right)^{\frac{1}{b}} \\
& \leq \frac{2 C}{C_{1}}\|f\|_{L^{A}(v ; \Omega)} v\left(S\left(C_{0}\right)\right)^{\frac{1}{b}} v(\Omega)^{\frac{1}{b\left(\sigma^{\prime} / b\right)^{\prime}}+\frac{2 \sigma-b}{\sigma}}=\frac{C}{\tau_{0}\left(1-2^{-\epsilon}\right)} v\left(S\left(C_{0}\right)\right)^{\frac{1}{b}} .
\end{aligned}
$$

Hence,

$$
v\left(S_{0}\right) \leq\left(\frac{C}{\tau_{0}\left(1-2^{-\epsilon}\right)}\right)^{b},
$$

and so we can choose $\tau_{0}>0$ independent of both $\mathbf{u}, f$ such that

$$
\mu_{0}=v\left(S\left(C_{0}\right)\right)<e^{-2}, \quad \tau_{0} \geq \max \left\{\frac{e C}{1-2^{-\epsilon}}, \frac{e C}{\epsilon}\right\}
$$


where $C$ is as in (4.9). We may now proceed exactly as in the proof of (3.6) to get that (4.10) holds. This completes our proof.

\section{TheOrem 1.7 IS ALMOST SHARP}

In this section we construct Example 1.9 that shows that Theorem 1.7 is almost sharp in the case of the Laplacian. Our example is intuitively straightforward. Let our domain $\Omega \subset \mathbb{R}^{n}, n \geq 3$, be the unit ball $B=B(0,1)$, and define

$$
f(x)=|x|^{-2} \log \left(e+|x|^{-1}\right)^{-1} .
$$

Let $A(t)=t^{\frac{n}{2}} \log (e+t)^{q}$. We will show that $f \in L^{A}(B)$ if and only if $q<\frac{n}{2}-1$. Moreover, we claim that, at least formally, if $u$ is the solution of $\Delta u=f$ on $B$, then $u(0)=\infty$. For if we use the well-known fact that the Green's function for the unit ball is $c_{n}|x|^{2-n}$, then

$$
u(0)=c_{n} \int_{B}|x|^{-n} \log \left(e+|x|^{-1}\right)^{-1} d x=\infty .
$$

To make this argument rigorous we must justify our use of Green's formula which requires that the function $f$ be continuous on $B$. To overcome this, we give an approximation argument and show that the inequality

$$
\|u\|_{L^{\infty}(B)} \leq C\|f\|_{L^{A}(B)}
$$

cannot hold with a uniform constant. For each $k \geq 1$, let $\chi_{k}$ be a continuous, non-negative, radial function such that $\chi_{k}(x)=0$ if $|x| \leq 2^{-k-1}$, and $\chi_{k}(x)=1$ if $2^{-k} \leq x<1$. Define $f_{k}=u_{k}$. Each $f_{k}$ is continuous, and if $u_{k}$ is the solution to the Dirichlet problem

$$
\begin{cases}\Delta u_{k}=f_{k} & x \in B, \\ u_{k}=0 & x \in \partial B\end{cases}
$$

then at the origin it is given by

$$
u_{k}(0)=c_{n} \int_{B}|x|^{2-n} f_{k}(x) d x \geq c_{n} \int_{2^{-k} \leq|x|<1}|x|^{-n} \log \left(e+|x|^{-1}\right)^{-1} d x .
$$

It is immediate that $u_{k}(0) \rightarrow \infty$ as $k \rightarrow \infty$. Since by monotonicity of the norm, $\left\|f_{k}\right\|_{L^{A}(B)} \leq\|f\|_{L^{A}(B)}$, we have that the inequality

$$
u_{k}(0) \leq\left\|u_{k}\right\|_{L^{\infty}(B)} \leq C\left\|f_{k}\right\|_{L^{A}(B)} \leq C\|f\|_{L^{A}(B)}
$$

cannot hold with a uniform constant if $f \in L^{A}(B)$.

Therefore, to complete the proof, it will suffice to show $f \in L^{A}(B)$ if and only if $q<\frac{n}{2}-1$. By the definition of the Luxemburg norm, it will suffice to show that $f(A) \in L^{1}(B)$. But this is straightforward:

$$
A(f(x))=f(x)^{\frac{n}{2}} \log (e+f(x))^{q}
$$




$$
\begin{aligned}
& =x^{-n} \log \left(e+|x|^{-1}\right)^{-\frac{n}{2}} \log \left(e+|x|^{-2} \log \left(e+|x|^{-1}\right)^{-1}\right)^{q} \\
& \approx x^{-n} \log \left(e+|x|^{-1}\right)^{-\frac{n}{2}} \log \left(e+|x|^{-1}\right)^{q}
\end{aligned}
$$

where the implicit constant only depends on $q$. Thus, $A(f) \in L^{1}(B)$ if and only if $\frac{n}{2}-q>1$, or equivalently, $q<\frac{n}{2}-1$.

\section{REFERENCES}

[1] S Chanillo and R. L. Wheeden. Weighted Poincaré and Sobolev inequalities and estimates for weighted Peano maximal functions. Amer. J. Math., 107(5):1191-1226, 1985.

[2] M Christ. Hypoellipticity in the infinitely degenerate regime, complex analysis and geometry (Columbus, OH, 1999). Ohio State Univ. Math. Res. Inst. Publ. de Gruyter, Berlin, 9:59-84, 2001.

[3] S.-K. Chua and R. L. Wheeden. Existence of weak solutions to degenerate $p$-Laplacian equations and integral formulas. J. Differential Equations, 263(12):8186-8228, 2017.

[4] A. Cianchi. Strong and weak type inequalities for some classical operators in Orlicz spaces. $J$. London Math. Soc. (2), 60(1):187-202, 1999.

[5] D. Cruz-Uribe, J. M. Martell, and C. Pérez. Weights, extrapolation and the theory of Rubio de Francia, volume 215 of Operator Theory: Advances and Applications. Birkhäuser/Springer Basel AG, Basel, 2011.

[6] D. Cruz-Uribe, S. Rodney, and E. Rosta. Poincaré inequalities and Neumann problems for the p-Laplacian. Canad. Math. Bull., 61(4):738-753, 2018.

[7] D. Cruz-Uribe, S. Rodney, and E. Rosta. Global Sobolev inequalities and degenerate $p$ Laplacian equations. J. Differential Equations, 268(10):6189-6210, 2020.

[8] E. De Giorgi. Sulla differenziabilità e l'analiticità delle estremali degli integrali multipli regolari. Mem. Accad. Sci. Torino. Cl. Sci. Fis. Math. Nat., 3:25-43, 1957.

[9] L. C. Evans and R. F. Gariepy. Measure theory and fine properties of functions. Textbooks in Mathematics. CRC Press, Boca Raton, FL, revised edition, 2015.

[10] E. B. Fabes, C. E. Kenig, and R. P. Serapioni. The local regularity of solutions of degenerate elliptic equations. Comm. Partial Differential Equations, 7(1):77-116, 1982.

[11] G. B. Folland. Real analysis. Pure and Applied Mathematics (New York). John Wiley \& Sons, Inc., New York, second edition, 1999. Modern techniques and their applications, A WileyInterscience Publication.

[12] D. Gilbarg and N. S. Trudinger. Elliptic Partial Differential Equations of Second Order. Classics in Mathematics. Springer-Verlag, Berlin, 2001. Reprint of the 1998 edition.

[13] L. Korobenko, C. Rios, E. T. Sawyer, and R. Shen. Sharp local boundedness and maximum principle in the infinitely degenerate regime via De Giorgi iteration. Memoirs Amer. Math. Soc., to appear. arXiv1608.01630v4.

[14] M. A. Krasnosel'skiı̌ and Ja. B. Rutickiǔ. Convex functions and Orlicz spaces. Translated from the first Russian edition by Leo F. Boron. P. Noordhoff Ltd., Groningen, 1961.

[15] M. T. Lacey and S. Spencer. On entropy bumps for Calderón-Zygmund operators. Concr. Oper., 2(1):47-52, 2015.

[16] V. G. Maz'ya. Some estimates of solutions of second-order elliptic equations. Dokl. Akad. Nauk SSSR, 137:1057-1059, 1961.

[17] V. G. Maz'ya. Weak solutions of the Dirichlet and Neumann problems. Trudy Moskov. Mat. Obšč., 20:137-172, 1969. 
[18] D. D. Monticelli and S. Rodney. Existence and spectral theory for weak solutions of Neumann and Dirichlet problems for linear degenerate elliptic operators with rough coefficients. $J$. Differential Equations, 259(8):4009-4044, 2015.

[19] D. D. Monticelli, S. Rodney, and R. L. Wheeden. Boundedness of weak solutions of degenerate quasilinear equations with rough coefficients. Differential and Integral Equations, 25(1-2):143$200,2012$.

[20] M. M. Rao and Z. D. Ren. Theory of Orlicz spaces, volume 146 of Monographs and Textbooks in Pure and Applied Mathematics. Marcel Dekker Inc., New York, 1991.

[21] S. Rodney. Existence of weak solutions of linear subelliptic Dirichlet problems with rough coefficients. Canad. J. Math., 64(6):1395-1414, 2012.

[22] E. T. Sawyer and R. L. Wheeden. Degenerate Sobolev spaces and regularity of subelliptic equations. Trans. Amer. Math. Soc., 362(4):1869-1906, 2010.

[23] G. Stampacchia. Le problème de Dirichlet pour les équations elliptiques du second ordre à coefficients discontinus. Ann. Inst. Fourier (Grenoble), 15(fasc. 1):189-258, 1965.

[24] S. Treil and A. Volberg. Entropy conditions in two weight inequalities for singular integral operators. Adv. Math., 301:499-548, 2016.

[25] N. S. Trudinger. Linear elliptic operators with measurable coefficients. Ann. Scuola Norm. Sup. Pisa Cl. Sci. (3), 27:265-308, 1973.

[26] X. Xu. Logarithmic up bounds for solutions of elliptic partial differential equations. Proc. Amer. Math. Soc., 139(10):3485-3490, 2011.

David Cruz-Uribe, OfS, Dept. of Mathematics, University of Alabama, Tuscaloosa, AL 35487, USA

Email address: dcruzuribe@ua.edu

Scott Rodney, Dept. of Mathematics, Physics and Geology, Cape Breton UniverSITY, Sydney, NS B1Y3V3, CA

Email address: scott_rodney@cbu.ca 